\title{
A Simulation Study OF IP Switching
}

\author{
Steven Lin and Nick McKeown \\ Department of Electrical Engineering \\ Stanford University \\ Stanford, CA 94305 \\ sclin@leland.stanford.edu nickm@ee.stanford.edu
}

\begin{abstract}
Recently there has been much interest in combining the speed of layer- 2 switching with the features of layer-3 routing. This has been prompted by numerous proposals, including: IP Switching [1], Tag Switching [2], ARIS [3], CSR [4], and IP over ATM [5]. In this paper, we study IP Switching and evaluate the performance claims made by Newman et al in [1] and [6]. In particular, using ten network traces, we study how well IP Switching performs with traffic found in campus, corporate, and Internet Service Provider (ISP) environments. Our main finding is that IP Switching will lead to a high proportion of datagrams that are switched; over $75 \%$ in all of the environments we studied. We also investigate the effects that different flow classifiers and various timer values have on performance, and note that some choices can result in a large VC space requirement. Finally, we present recommendations for the flow classifier and timer values, as a function of the VC space of the switch and the network environment being served.
\end{abstract}

\section{What is IP Switching?}

The Internet is overwhelmed with traffic, with user demand for bandwidth well in excess of supply. This should come as no great surprise - the number of users of the Internet has grown exponentially for ten straight years, with each user adding more demand to the network. Over time, each user's traffic tends to increase, adding further to the demand. And at the same time, a larger proportion of traffic traverses multiple domains, swamping campus backbones, corporate intranets, and the core of the Internet. In response to the demand, network equipment vendors and service providers have developed and deployed faster and faster routers. Yet demand for bandwidth far outstrips the current link and switching capacity of the Internet.

The performance of network routers is usually limited by two main tasks: (1) Examining the destination address of incoming datagrams, indexing into a routing table to determine the next hop, and (2) copying incoming datagrams to their outgoing interface. The routing lookup is not straightforward; the router must find the longest prefix match over a space of approximately $2^{31}$ possible addresses. It is unusual for a router today to perform more than 100,000 address lookups per second. Perhaps surprisingly, the performance of most current routers is limited by their ability to copy data between interfaces. This is because datagrams frequently traverse shared busses multiple times before reaching their outgoing interface.

A variety of techniques have been proposed to accelerate routing lookups [7], and replace shared busses with switched back-

Work supported by the Center for Integrated Systems at Stanford University. planes [8], [9].

Alternative techniques have been proposed that replace longest prefix routing lookups with simple exact matching, and use layer-2 switches to perform fast data copying. We generically call these techniques "label swapping"; they include IP Switching [1], CSR [4], IP over ATM [5], ARIS [3], and Tag Switching [2].

IP Switching integrates IP routing with fast lookup and copying mechanisms of an ATM switch ${ }^{1}$. Based on IFMP (Ipsilon Flow Management Protocol) [10] [11], IP Switching has received a lot of attention. The CSR proposal is similar to IP Switching in many respects, so our findings generally apply to the CSR architecture. On the other hand, ARIS and Tag Switching are very different, so our results do not represent the performance of these label swapping systems.

Consider the IFMP-speaking IP Switch shown in Figure 1. We assume that the switch is surrounded by a number of identical neighbors. Our switch begins by establishing adjacency with its neighbors, identifying them as fellow IFMP switches. IP datagrams arrive at the switch encapsulated into AAL5 frames and segmented into ATM cells. These datagrams are part of a flow between two user processes, or between two end points in the network. Initially, all datagrams from all flows are received and transmitted on a single, default ATM virtual circuit (VC). Our switch attempts to identify flows, classifying them according to their expected duration. If the switch decides that the flow will be long lasting, it will attempt to establish a unique $\mathrm{VC}$ for the flow, so that it may be switched quickly in hardware. The switch tells its upstream neighbor to begin sending all datagrams that are in that flow on a separate VC. This is achieved by sending an IFMP redirect message to the next switch that is upstream in the flow. Similarly,

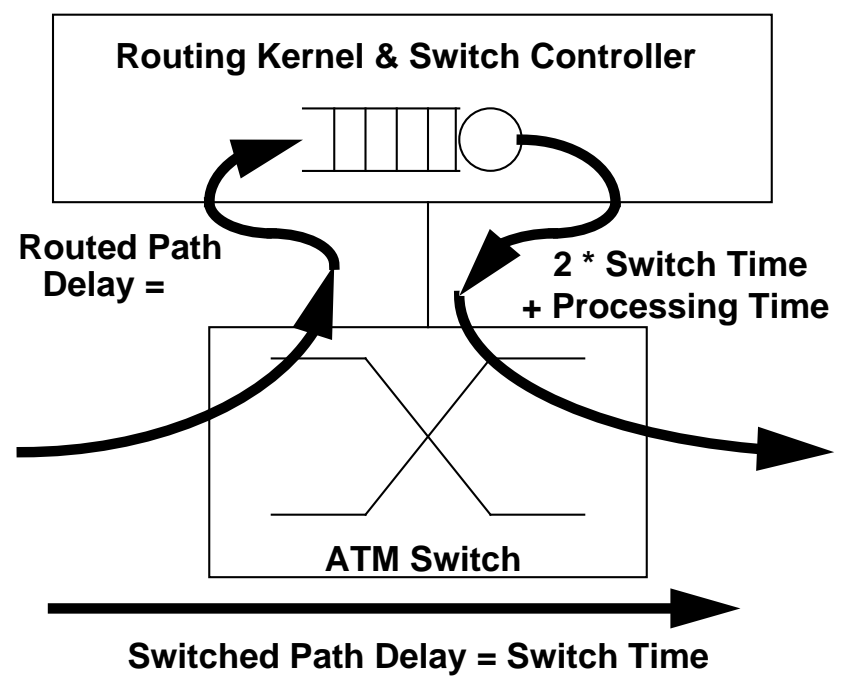

Figure 1: Simple Block Diagram of IP Switch

${ }^{1}$ Although IP Switching could be used on any layer- 2 switch, for the remainder of this paper we only consider ATM, since this is what has been used in practice. 
our switch will receive a redirect message from its downstream neighbor when the downstream neighbor detects a flow. We can think of the redirect messages as binding the flow to an ATM virtual circuit. When the flow has been successfully bound, our switch can perform hardware layer-2 switching from the incoming $\mathrm{VC}$ to the outgoing $\mathrm{VC}$, without consulting any routing tables or performing any other processing.

This is shown in Figure 1, where the first datagrams in a flow must traverse the 'slow path' through a conventional IP router. The router reassembles cells into AAL5 frames, removes the IP datagram, and makes a routing decision. The datagram is encapsulated and segmented into cells before being sent on its way. In addition, a packet classifier attempts to recognize flows and estimate whether they are worth binding to an ATM VC. If so, a redirect message is sent to the upstream switch, and future datagrams will travel along the fast path. Higher performance is achieved if a large proportion of the datagrams are able to take the fast path. For this reason, we use the percentage of datagrams switched as the primary metric in our study.

\section{Flow Classification}

Each IP Switch contains a flow classifier: an entity that identifies flows and decides whether or not they should be bound to an ATM VC. IFMP defines two types of flow: (1) Flow Type 1: a flow of IP datagrams between two processes. In other words, datagrams that all contain the same values for the following IP and transport fields: IP version, source and destination addresses, header length, type-of-service, time-to-live (TTL), protocol, and transport port numbers. (2) Flow Type 2: a flow of datagrams between two IP addresses. All the datagrams in a type 2 flow contain the same IP version, source and destination addresses, header length, and TTL.

An IP Switch must have a policy to determine when a flow will be bound to an ATM VC. This policy is outside the scope of the IFMP protocol description, but must be present in any IFMP implementation. The IP Switch designer may choose from a variety of techniques to determine if a flow should be bound to a VC. On one hand, it is clearly desirable to bind as many flows as possible, because this will enable a large number of datagrams to be switched at layer-2, reducing the number of layer-3 routing decisions. On the other hand, if short-lived flows, or flows with a small number of datagrams are switched, this will waste the VC space of the switch. It will also lead to a large number of redirect messages.

Our simulation uses three types of flow classifier. Each classifier is designed to switch a large number of datagrams at layer-2, while wasting a small number of VCs, and sending as few redundant redirect messages as possible. The first classifier is the $X / Y$ Classifier. This classifier watches until it sees $\mathrm{X}$ datagrams within a Y second window that all match a specific flow. The switch then binds the flow to an ATM VC, switching any future datagrams that meet that flow specification. The motivation behind the X/Y Classifier is that if there have already been $\mathrm{X}$ datagrams from a flow in $\mathrm{Y}$ seconds then it is reasonable to expect that there will be more datagrams from this flow in a short amount of time. The only remaining problem is to determine values for $\mathrm{X}$ and $\mathrm{Y}$.

The second classifier is the Protocol Classifier, which simply assigns all TCP packets to flows. The rationale for this classifier is that connection-based communications generally last longer and have more datagrams sent over a short time than connectionlessbased communications.

Finally, the Port Classifier uses the transport-layer port numbers to decide which flows to bind. In our study, we decided to bind all TCP flows going to or from the following ports: ftp-data (20), telnet (23), smtp (25), http (80), and nntp (119). The goal is to select those applications which tend to generate long-lasting flows, and/or flows which contain a large number of datagrams. It could be argued that additional or alternative services/ports be used instead of the ones above; these were picked because they made up the largest percentage of TCP packets seen in the traces we analyzed.

\section{Delays in Flow Establishment}

There are also several delays associated with the creation and deletion of flows, with values unspecified in the IFMP description. These delays are either chosen or will occur naturally in any implementation. The following delays were modeled by our simulation:

Flow Creation Delay: In practice, there is a delay between the time a flow is detected, and the time at which the upstream node begins sending datagrams on the new VC. This delay is due to the time required to form the redirect message, its propagation delay upstream, and the processing time of the message at the upstream node. We model this delay as a constant. Note that this delay is not directly related to the maximum connection establishment rate of the IP Switch; we do not have to wait for one connection to be fully established before beginning to establish another.

Flow Deletion Delay: There must be some decision made as to when to delete a flow. According to the IFMP specification, the upstream neighbor will time-out a flow if it does not receive additional redirect messages. To prevent flows from timing out prematurely, a switch sends redirect messages upstream at regular intervals. In our simulation, we approximate the time-out by deleting a flow after some constant number of seconds of inactivity.

\section{Questions about IP Switching Perfor- mance}

In our study, we set out to answer six main questions about the performance of IP Switching:

1) Are the claims of switching performance presented by the inventors of IP Switching valid?

2) To work effectively, does IP Switching require an exceptionally large VC space?

3) How do the flow creation delay and flow deletion delay affect performance?

4) Which flow classifiers work best in different environments?

5) How does the speed of the router affect performance and datagram misordering?

6) Given a fixed VC Space and knowledge about the environment in which the IP Switch will be used, how should parameters be chosen to provide the best performance?

\section{Experimental Setup / Procedure}

Ten network traces were analyzed by a high-level simulation of an IP Switch. Six of the traces (DEC-PKT-1 through DEC-PKT4, LBL-PKT-4 and -5) are each one hour in length and were taken on corporate gateways to the Internet. These traces are freely available from the Internet Traffic Archive ${ }^{2}$. Two more, shorter traces were taken at the FIXWEST west coast federal interexchange node, and were obtained from the National Laboratory for Applied Network Research (NLANR) ${ }^{3}$. Finally, the last two traces were extracted from a four-day long trace of two campus backbone rings at

\footnotetext{
${ }^{2}$ Internet Traffic Archive traces are available at http:// town.hall.org/Archives/pub/ITA/html/traces.html ${ }^{3}$ FIXWEST/NLANR traces are available at ftp:// oceana.nlanr.net/Traces/FR+/. Also see the NLANR home page at http://www.nlanr.net for more information.
} 


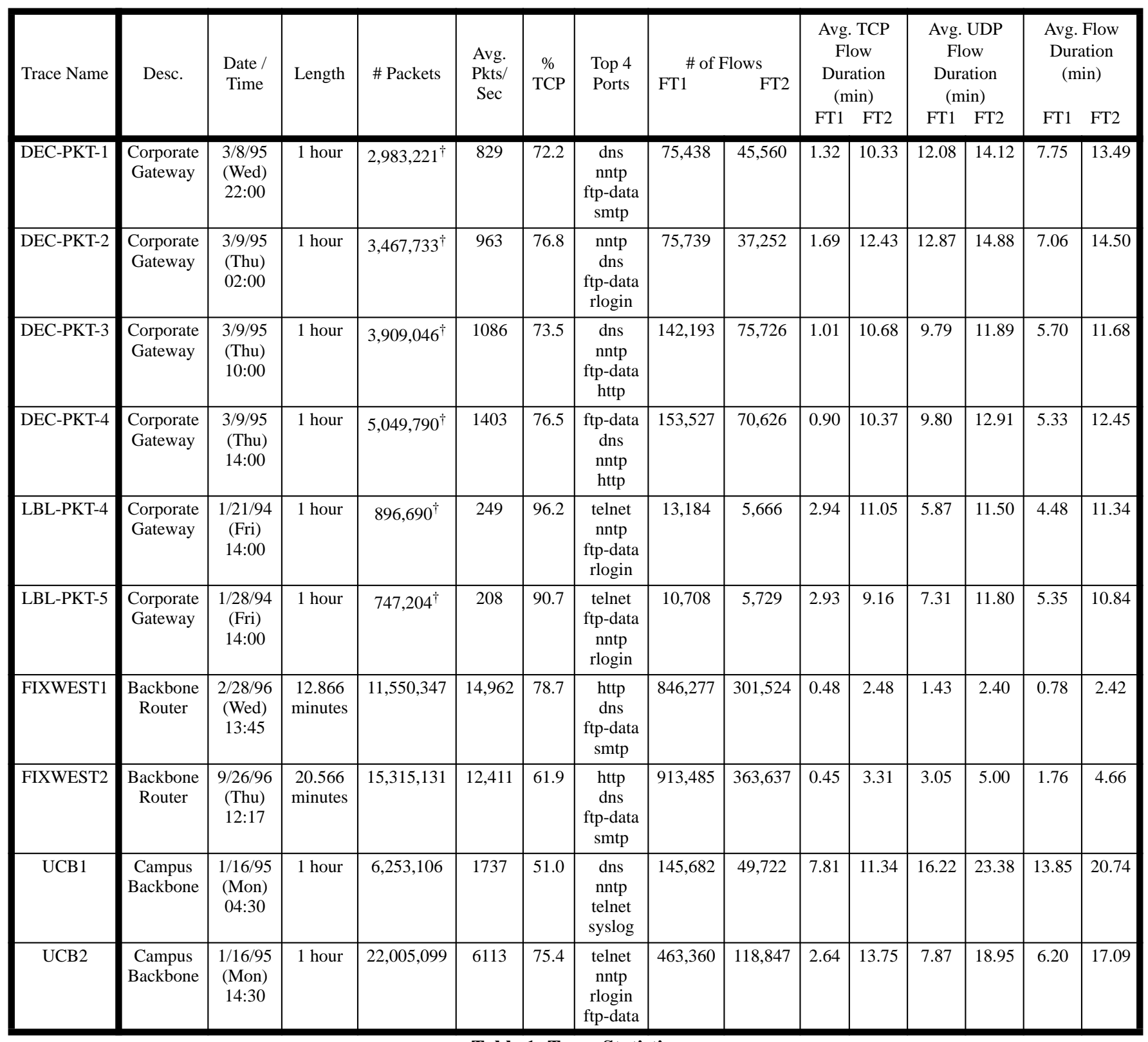

Table 1: Trace Statistics

These numbers reflect those packets analyzed in the simulation and include all TCP and UDP packets. There were other packets in the raw traces, but not enough information associated with these other packets to analyze them.

the University of California, Berkeley ${ }^{4}$. The two hours examined were the most busy and least busy hours (in terms of packet arrival rate) throughout all 4 days.

Some statistics from the traces are shown in Table 1. The "number of packets" column represents the number of datagrams actually processed by the simulator and includes all TCP and UDP datagrams. The "\% TCP" column shown the percentage of IP datagrams with the protocol field set to TCP. The "top 4 ports" column shows, in order, the four most popular source and destination ports for TCP and UDP. The "\# of flows" column shows the number of flows of type 1 and flows of type 2. Finally, the "Average Flow Duration" columns give the average lifetime of TCP flows,
UDP flows, and all flows, using the flow type 1 and 2 flow definitions. Because the traces were finite duration, the flow duration numbers are approximate, as some flows were started before the sample time, and some lasted beyond the sample time.

Our simulation modeled a single IP switch port. The traces were used as input to this port, and we were able to vary the VC space of the port, the flow creation and deletion delays, the flow type to use, and the flow classifier. Results generated by the simulation include percentage of datagrams switched (vs. those forwarded by the routing kernel), and maximum number of VCs used during the simulation. The source code for the simulation is freely available via anonymous ftp at: ftp://klamath.stanford.edu/pub/ outgoing/issim.tar.gz.

\footnotetext{
${ }^{4}$ The UC Berkeley trace is currently unavailable.
} 


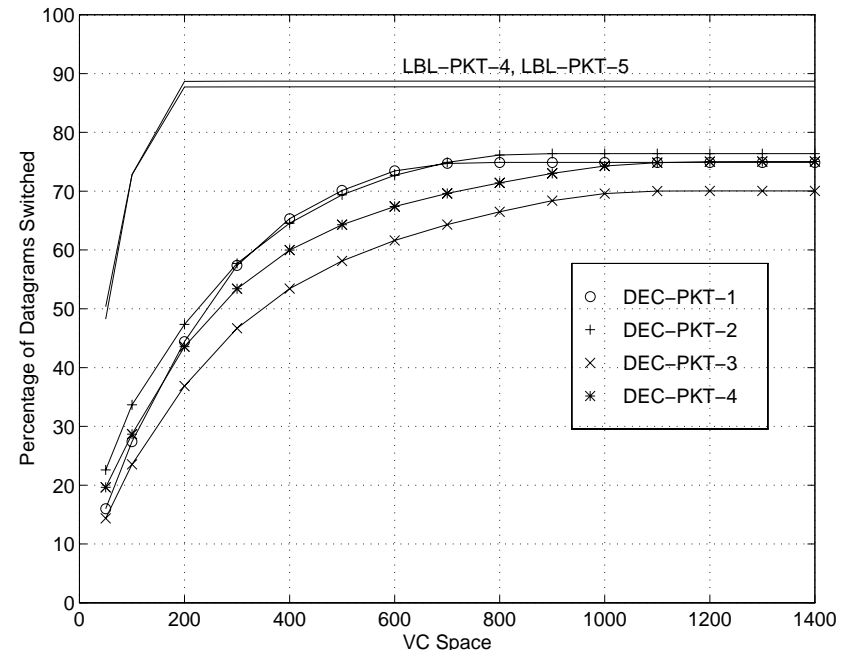

Figure 2: \% Datagrams Switched vs. VC Space; Corporate Traces, Flow Type 1

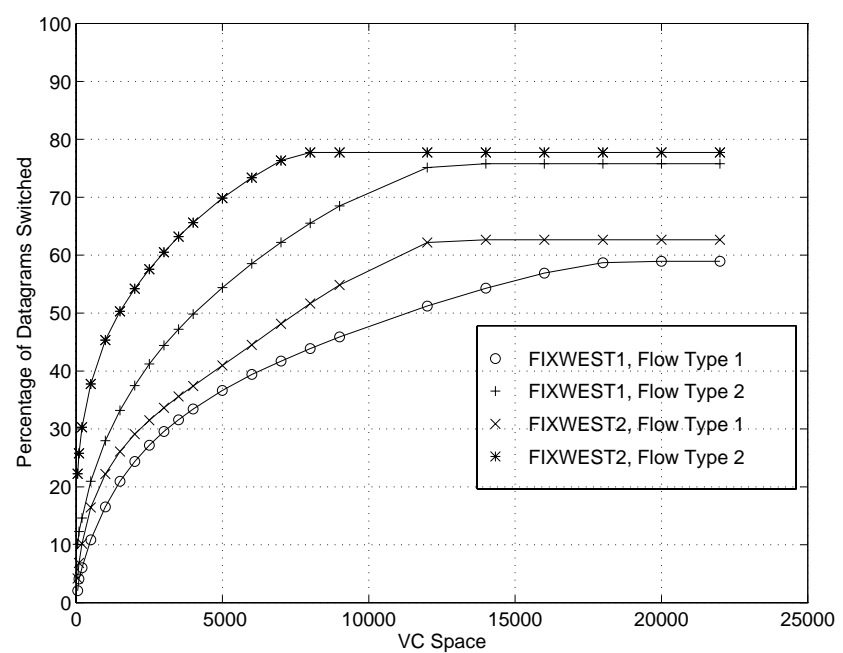

Figure 4: \% Datagrams Switched vs. VC Space; ISP Traces

\section{Results}

\section{Percentage of Datagrams Switched and VC Space Requirements:}

We begin by addressing our first two questions: Are the claims of switching performance presented by the inventors of IP Switching valid? And to work effectively, does IP Switching require an exceptionally large VC space?

Figures 2 through 5 show the results of a set of simulations in which the VC space of the switch port is varied. A new flow can only be bound to an ATM VC if there is at least one VC available. In these experiments, an $\mathrm{X} / \mathrm{Y}$ classifier was used with $\mathrm{X}=10$ packets and $Y=20$ seconds. From these plots, we can begin to answer our questions. First notice that in every trace, at least $75 \%$ of the datagrams are switched when flow type 2 is used (we will see later that this percentage can be slightly increased with a different choice of simulation parameters). This seems to corroborate the inventors' claim that about $80 \%$ of datagrams will be switched [1] [6]. The percentage is lower when flow type 1 is used; this is because a flow of type 2 contains multiple flows of type 1 . To be bound, each flow of type 1 must fulfill the $\mathrm{X} / \mathrm{Y}$ requirements of the classifier, whereas a flow of type 2 must fulfill these requirements only once.

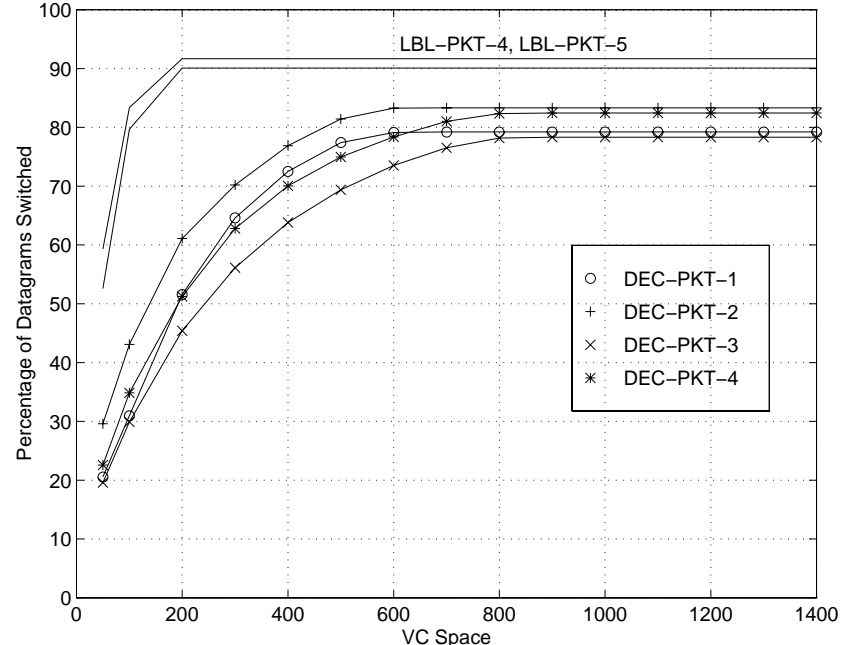

Figure 3: \% Datagrams Switched vs. VC Space; Corporate Traces, Flow Type 2

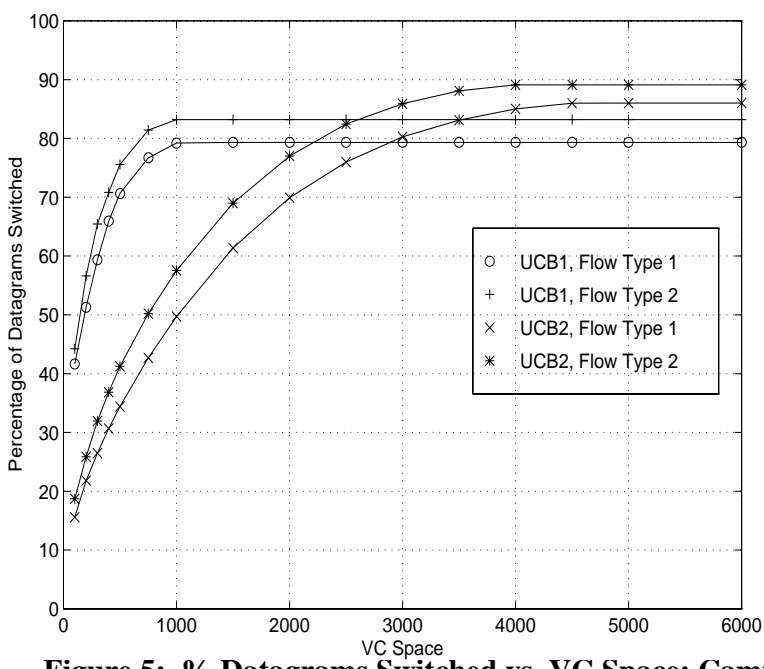

Figure 5: \% Datagrams Switched vs. VC Space; Campus Traces

Notice that for certain environments, namely at the corporate gateway and on the campus backbone, maximum performance can be reached with only a few thousand VCs. However, when the ISP traces are examined, it is apparent that about $10,000 \mathrm{VCs}$ are required to reach maximum performance. Although a high percentage of packets can still be switched, this VC usage is likely to increase with time and may become excessive for some hardware.

Finally, with the exception of the UCB traces, we notice that the behavior of similar traces is similar. For example, all four DEC traces exhibit similar behavior, as do both LBL traces. In order to clarify our results, the remainder of the plots feature only one representative plot from each data set, even though all nine traces were processed by the simulator.

\section{Flow Creation Delay:}

Next we try to answer our questions on delay: How do flow creation and deletion delays affect performance? We start by examining the sensitivity of performance to the flow creation delay.

As mentioned earlier, the flow creation delay is the time from when a redirect message is sent to the time at which datagrams begin to appear on the new VC. We model this delay as a constant, 


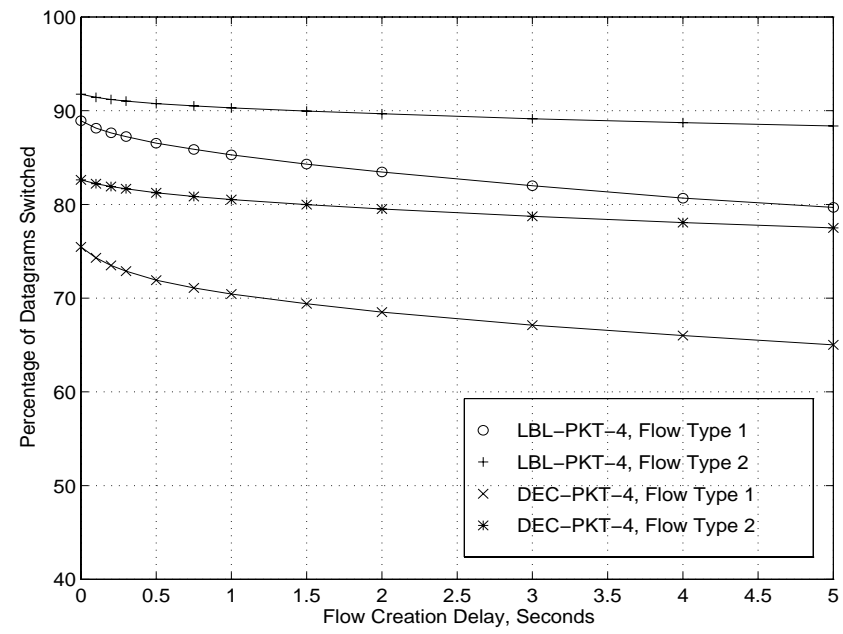

Figure 6: \% Datagrams Switched vs. Flow Creation Delay; Corporate Traces

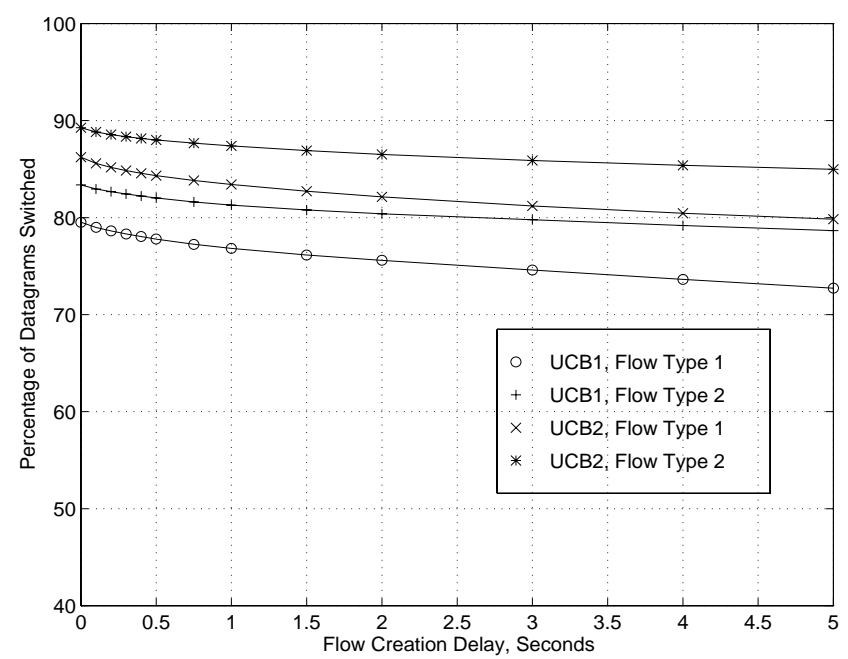

Figure 8: \% Datagrams Switched vs. Flow Creation Delay; Campus Traces

and see how performance is sensitive to its value. In all previous plots, this value was chosen to be $15 \mathrm{~ms}$.

As seen in Figures 6 through 8, the fraction of datagrams switched drops as the flow creation delay increases. This is expected; increasing the time to redirect a flow is equivalent to increasing the time to classify the flow. Both allow more datagrams to be forwarded through the routing path before hardware switching takes over. Because the ISP traces have a higher datagram arrival rate, they are affected more by this delay than the gateway and campus traces. Even at the maximum tested value of 5 seconds, however, the percentage of packets switched drops by less than $18 \%$ in all cases. In practice, the flow creation delay for an IP switch is on the order of 10-20 ms. For delays in this neighborhood, there is only a negligible effect on performance.

\section{Flow Deletion Delay:}

We now turn our attention to the flow deletion delay. This parameter is unspecified in the IFMP specification, but must be chosen by the implementor. There are conflicting goals in the selection of this value. The dilemma here is that a higher percentage of datagrams can be switched by being reluctant to delete flow bindings too early. However, VC space can be conserved by attempting to

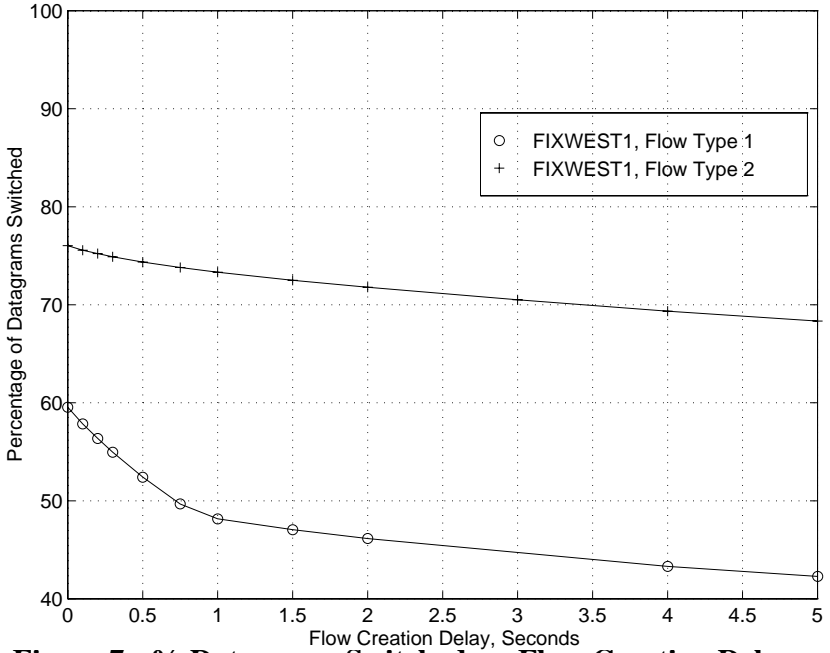

Figure 7: \% Datagrams Switched vs. Flow Creation Delay; ISP Traces

delete the flow binding (thus freeing a VC) as soon as possible.

From the simulation results in Figures 9 through 14, it seems that a fairly small flow timeout value ( 30 to 60 seconds) is sufficient to achieve close to maximum performance, while preventing an excessive waste of $\mathrm{VC}$ space. If, however, the selected deletion delay is too large, a switch can easily require over $32,000 \mathrm{VCs}$. In the Internet Service Provider environment where the traffic arrival rate is high, this can occur even at seemingly reasonable choices, such as 2 or $2 \frac{1}{2}$ minutes.

\section{Flow Classifier:}

Our next question is: Which flow classifier works best in different environments? All of the previous plots were made with an $\mathrm{X} / \mathrm{Y}$ classifier, with $\mathrm{X}=10$ and $\mathrm{Y}=20$. We start by seeing how changing $\mathrm{X}$ and $\mathrm{Y}$ affects the performance of the system. Figures 15 through 18 show results from one of the ISP traces as $\mathrm{X}$ is varied from 2 to 60 datagrams and $\mathrm{Y}$ is varied from 1 to 60 seconds. The other types of traces show similar trends, although with lower VC usage.

Using the extremely permissive classifier of " 2 packets in 1 minute", Figure 16 shows that as many as $90 \%$ of the datagrams qualify for switching. However, as shown in Figure 18, this performance is tempered by the extremely high VC usage. On the other hand, a strict classifier of "60 packets in 1 second" conserves VC space but makes it unlikely that a flow will be classified. Obviously, the best solution is somewhere between the two. It seems that the best choice depends on the VC space of the switch. If a large $\mathrm{VC}$ space is available, a more permissive classifier will produce better performance. However, if the VC space is limited, a permissive classifier will waste some VCs on less productive flows, and so a stricter classifier is a better choice. For example, if only 5000 VCs are available using flow type 2 in a FIXWEST-like environment, the "15 packets in 10 seconds" classifier, resulting in $69 \%$ packets switched, is a good compromise.

It should be pointed out that in the gateway and campus traces, even using the " 2 packets in 1 minute" classifier, no more than 11,000 VCs are ever used (and all classifiers with $X \geq 5$ packets require fewer than $6600 \mathrm{VCs}$ ). In other words, as before, for these types of environment, as long as the VC space is reasonably large, a permissive classifier may be used.

We also considered two other types of flow classifier: a Protocol Classifier and a Port Classifier. Although neither the protocol nor port fields appear in the flow type 2 descriptor, it is still possible 


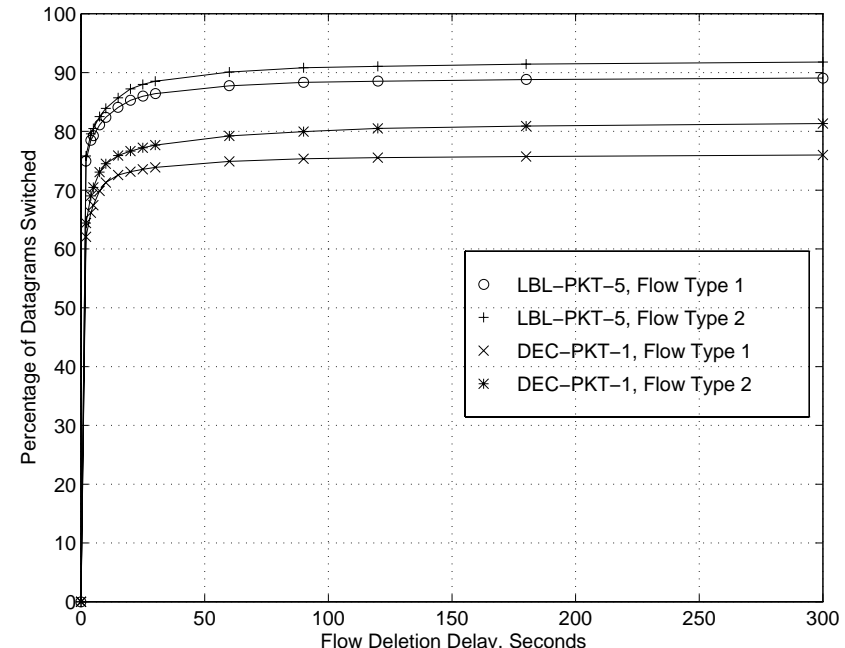

Figure 9: \% Datagrams Switched vs. Flow Deletion Delay; Corporate Traces

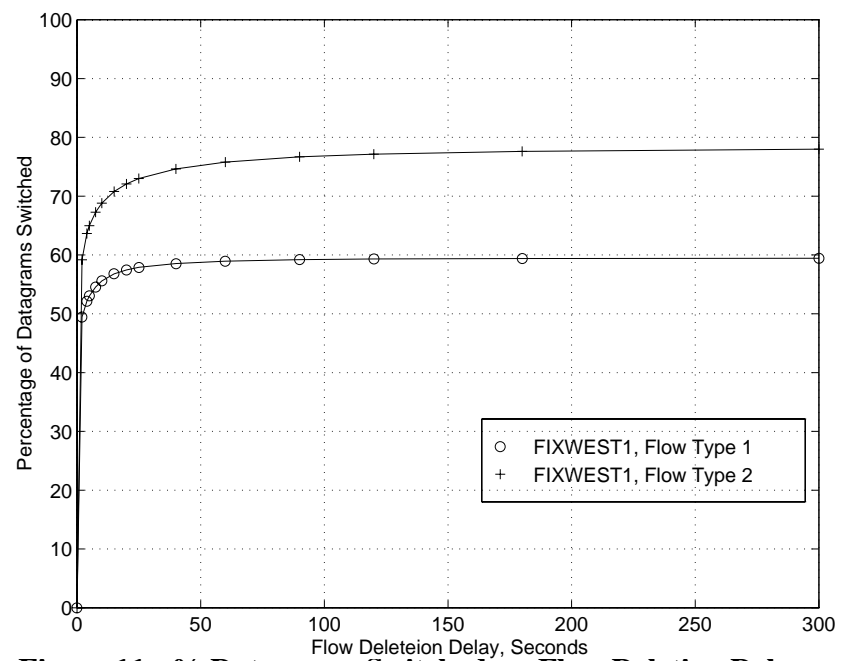

Figure 11: \% Datagrams Switched vs. Flow Deletion Delay; ISP Traces

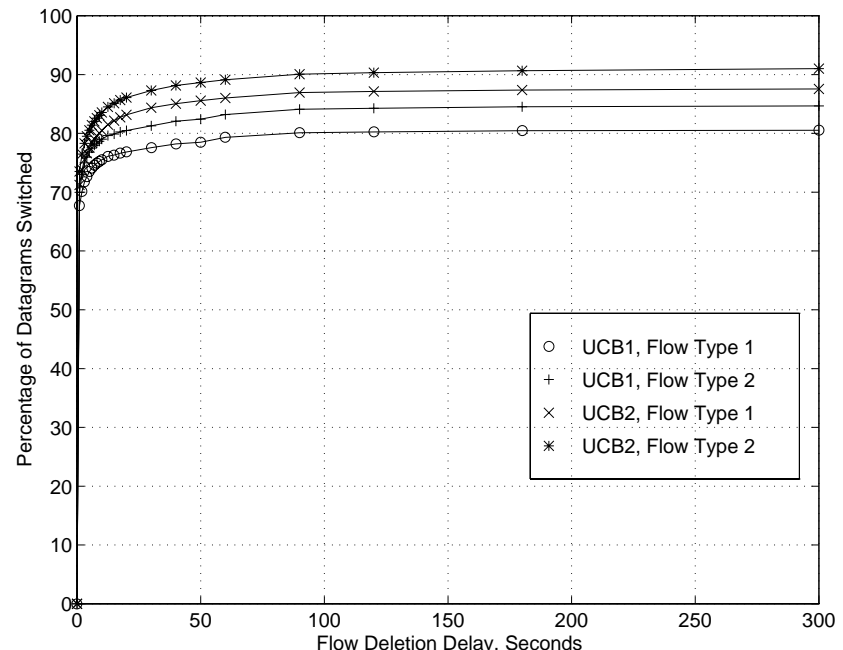

Figure 13: \% Datagrams Switched vs. Flow Deletion Delay; Campus Traces

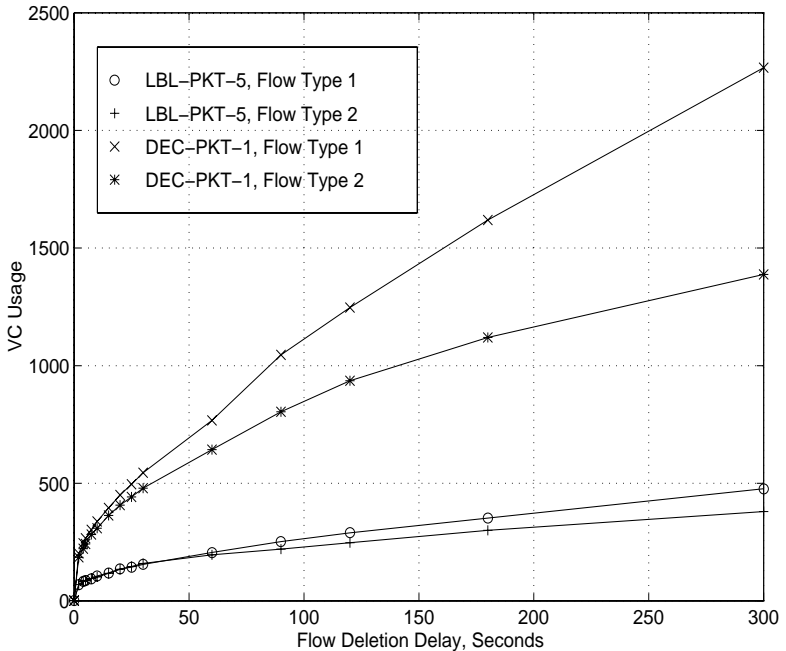

Figure 10: VC Usage vs. Flow Deletion Delay; Corporate Traces

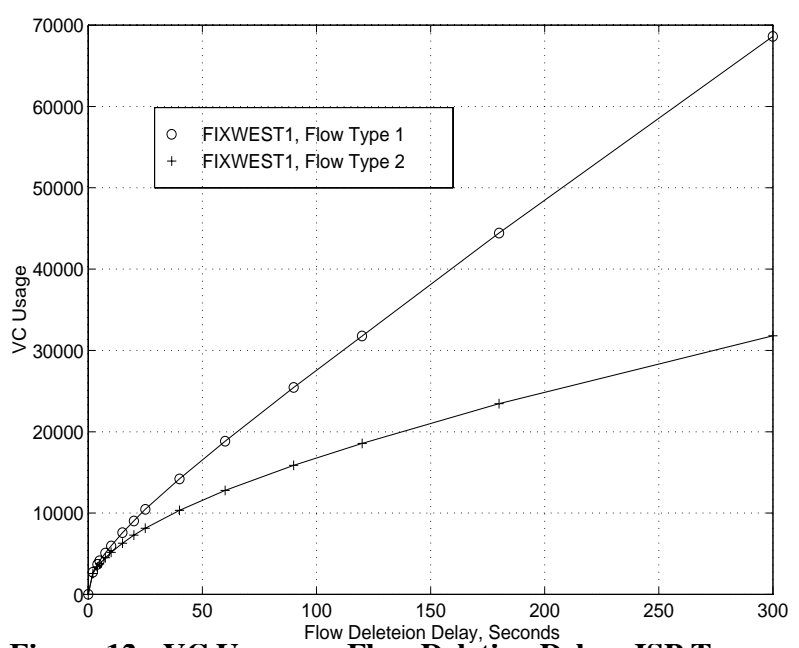

Figure 12: VC Usage vs. Flow Deletion Delay; ISP Traces

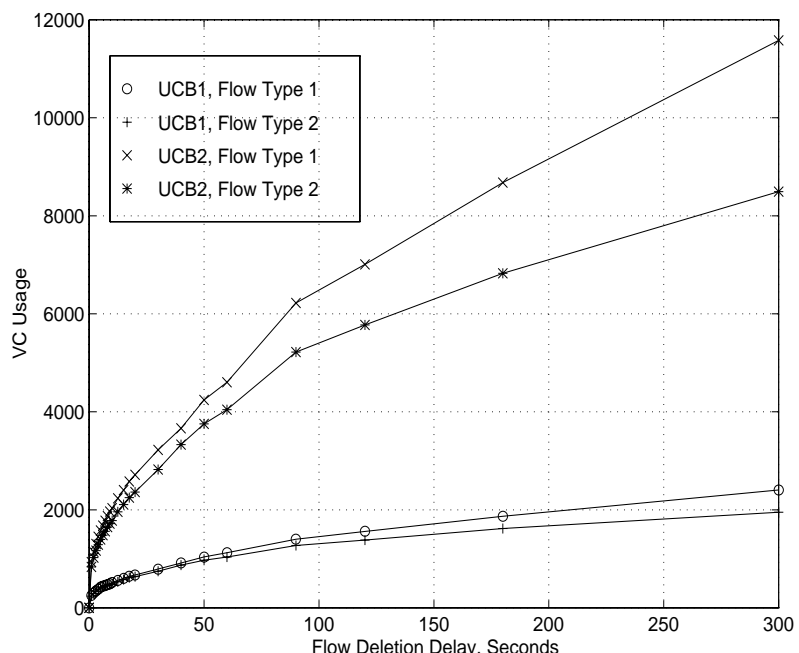

Figure 14: VC Usage vs. Flow Deletion Delay; Campus Traces 


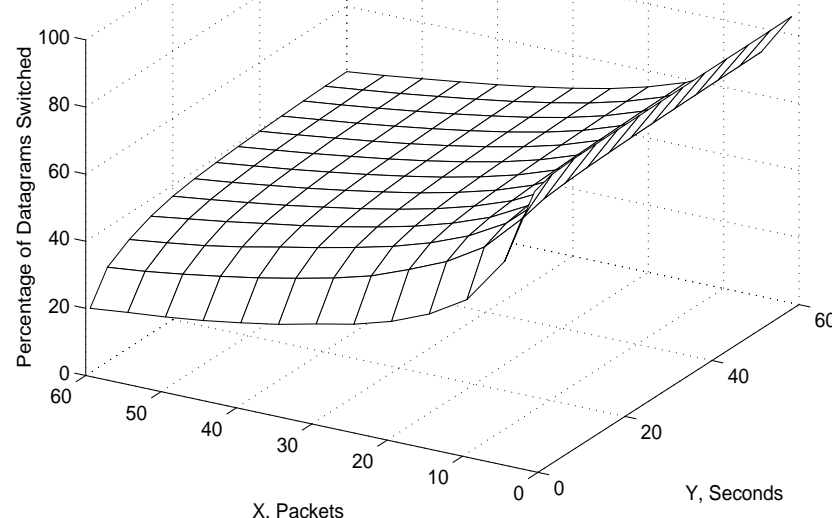

Figure 15: \% Datagrams Switched vs. X/Y Classifier; FIXWEST2, Flow Type 1

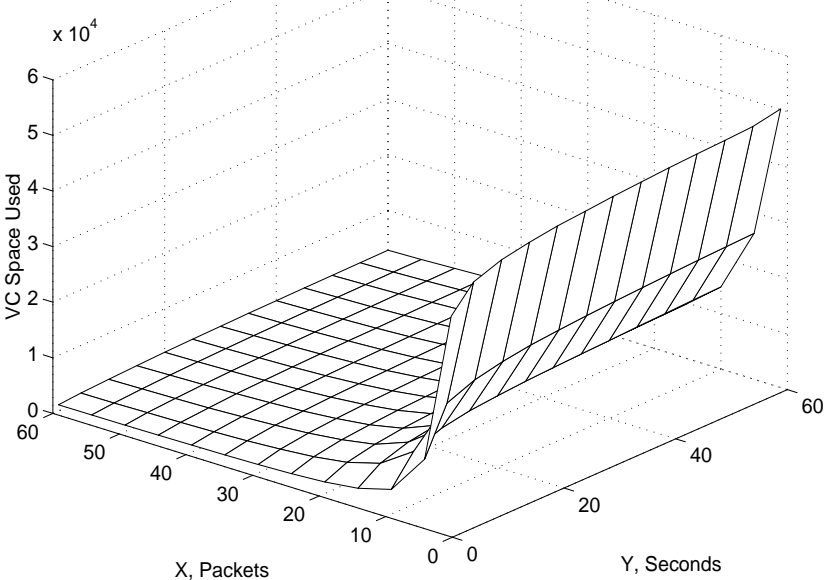

Figure 17: $\begin{aligned} & X, \text { Packets } \\ & \text { VC Usage vs. X/Y Classifier; FIXWEST2, Flow }\end{aligned}$ Type 1

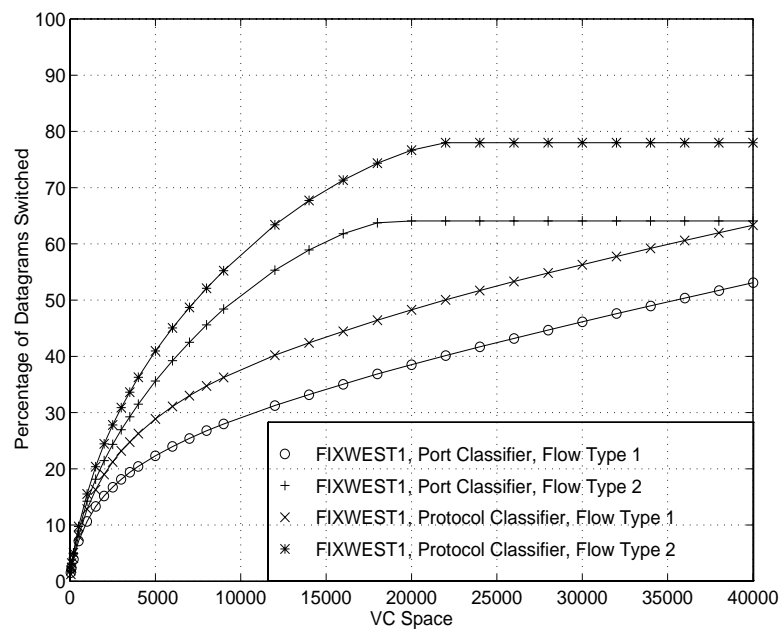

Figure 19: \% Datagrams Switched vs. VC Space

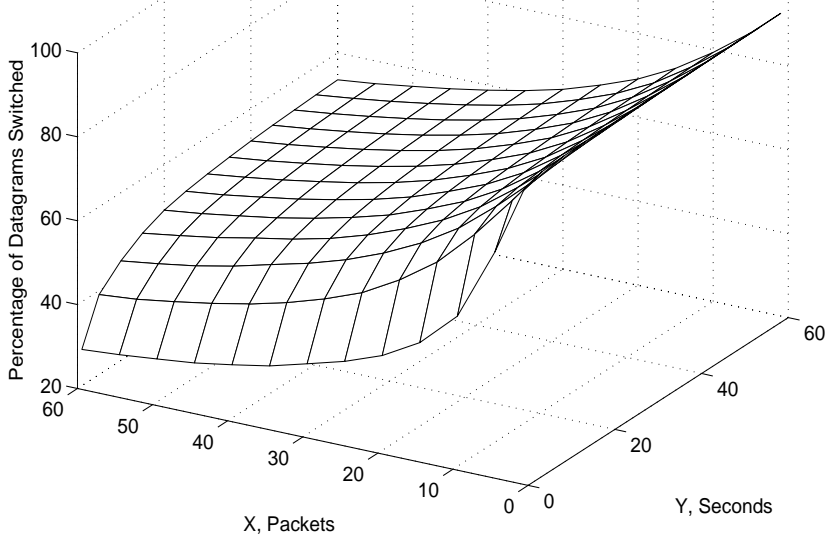

Figure 16: \% Datagrams Switched vs. X/Y Classifier; FIXWEST2, Flow Type 2

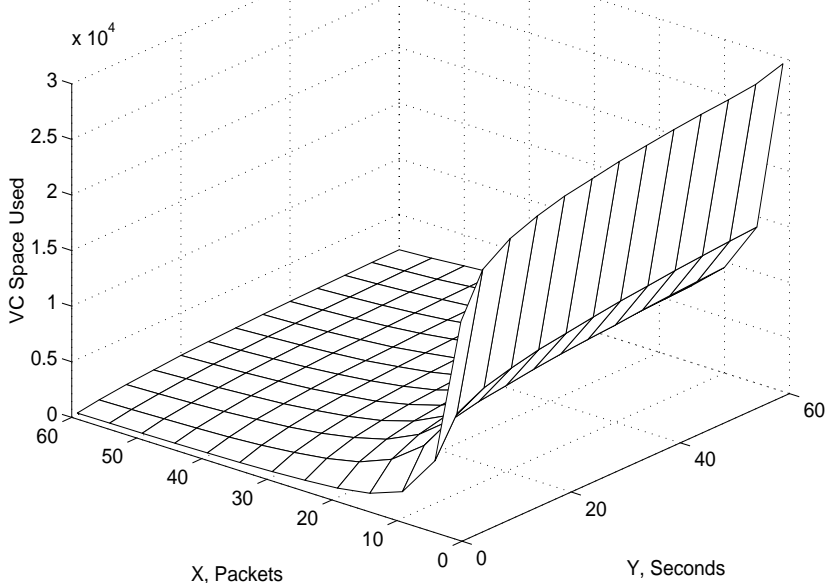

Figure 18: VC Usage vs. X/Y Classifier; FIXWEST2, Flow

Type 2

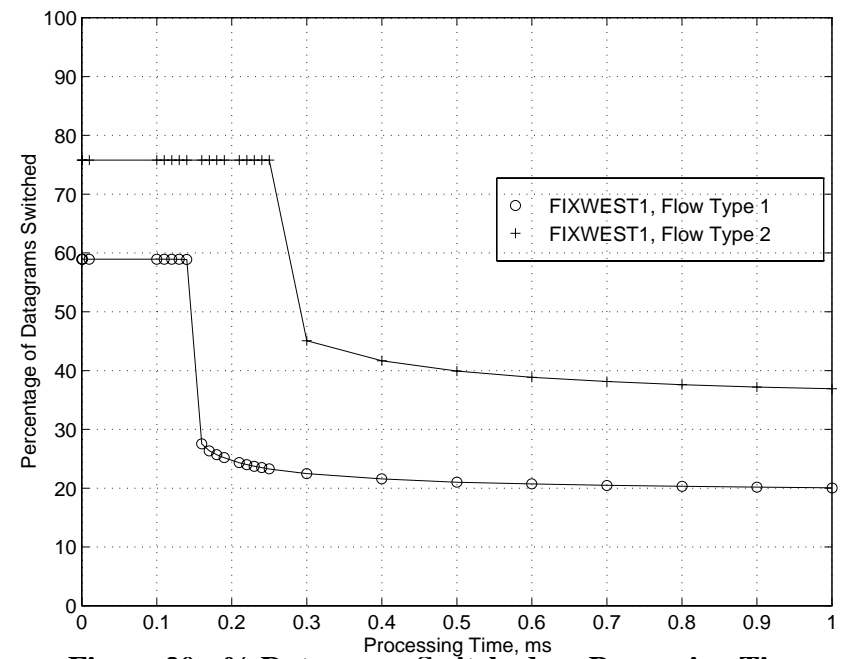

Figure 20: \% Datagrams Switched vs. Processing Time 


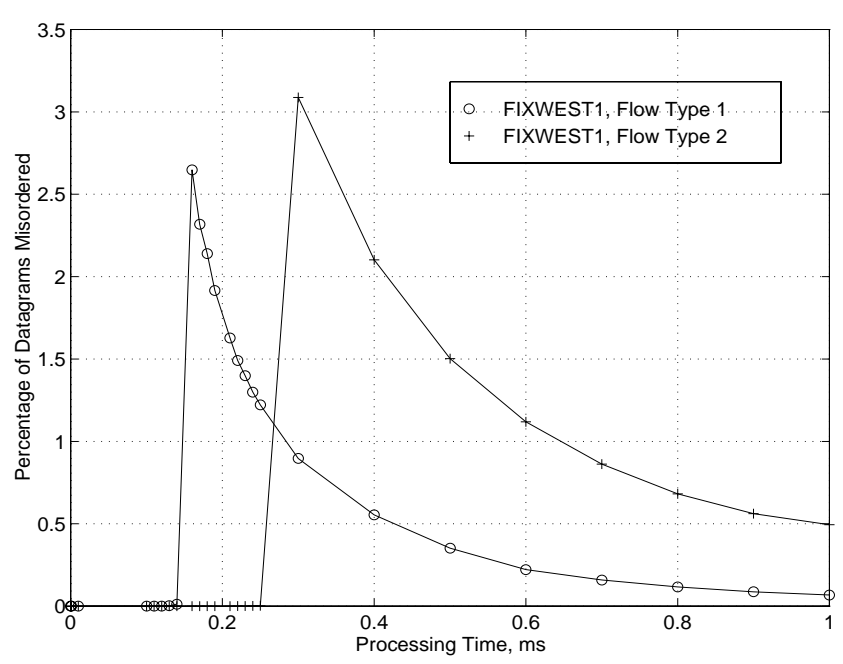

Figure 21: \% Datagrams Misordered vs. Processing Time

to use these fields for deciding when to establish a flow binding, but disregard the fields when deciding which flow a given datagram is part of.

Figure 19 shows the performance of these two classifiers on one of the ISP traces, as a function of the switch VC space. Although the Protocol classifier provides approximately the same maximum performance as the $\mathrm{X} / \mathrm{Y}$ classifier in terms of number of datagrams switched, it requires many more VCs than the $\mathrm{X} / \mathrm{Y}$ classifier to achieve this performance. The Port classifier is not able to achieve performance comparable to the $\mathrm{X} / \mathrm{Y}$ classifier, regardless of the VC space.

Because the classifier settings are dependent on the environment and switch capabilities, research is currently being done to investigate the use of dynamic classifier settings [14].

\section{Router Speed:}

Finally, we ask the question: How does the speed of the router/classifier affect performance and datagram misordering? As shown in Figure 1, incoming datagrams that are not part of a switched flow are placed onto a FIFO queue, where they wait to be processed $^{5}$. The classification process and the routing process occur simultaneously as datagrams are read from the queue. The speed of this processing, expressed as a fixed amount of time per datagram, was varied in our simulations. (Note that this processing time determines the router's "packet per second" capacity.)

Figure 20 shows the percentage of datagrams switched as a function of the router/classifier processing time. As this time increases, the percentage of datagrams switched remains fairly constant until it reaches a precipice, beyond which, it drops very rapidly. The precipice represents the processing time that causes the system to become unstable. When the routing time is too long, the queue of datagrams waiting to be routed grows without bound. Now, by the time a flow is classified, many of its constituent datagrams are already in the routing queue, so fewer datagrams are switched. In order to operate in a stable manner, the IP Switch must be able to route packets at a rate faster than this unstable point. For the Fixwest trace shown, using flow type 2, this unstable point occurs at a router processing time of $0.27 \mathrm{~ms}$, or a rate of about 3700 packets per second.

The other artifact that could be caused by a long processing time is the number of misordered datagrams. Looking back at Figure 1 , observe that before a flow is switched at layer-2, all packets travelling via the default VC must be switched once to enter the routing hardware, then processed, and switched again before leaving the IP Switch. It is possible that while some datagrams associated with the flow are being processed by the routing kernel,

${ }^{5}$ We set the switch latency to be 10 microseconds (approximately four ATM cell times at $155 \mathrm{Mbps}$ ).

\begin{tabular}{|c|c|c|c|c|c|c|c|c|c|c|}
\hline \multirow[t]{2}{*}{ Trace } & \multicolumn{2}{|c|}{$\begin{array}{c}\% \text { Datagrams } \\
\text { Switched }\end{array}$} & \multicolumn{2}{|c|}{$\%$ Bytes Switched } & \multicolumn{2}{|c|}{$\begin{array}{c}\text { Max. \# VC } \\
\text { Establishments/Sec }\end{array}$} & \multicolumn{2}{|c|}{$\begin{array}{c}\text { Max. \# VC } \\
\text { Teardowns/Sec. }\end{array}$} & \multicolumn{2}{|c|}{$\begin{array}{c}\text { Max. \# VC Events/ } \\
\text { sec }\end{array}$} \\
\hline & FT1 & FT2 & FT1 & FT2 & FT1 & FT2 & FT1 & FT2 & FT1 & FT2 \\
\hline DEC-PKT-1 & 84.5 & 87.6 & 86.9 & 88.7 & 28 & 17 & 30 & 18 & 46 & 28 \\
\hline DEC-PKT-2 & 85.5 & 90.7 & 90.9 & 93.6 & 33 & 15 & 38 & 16 & 51 & 22 \\
\hline DEC-PKT-3 & 79.0 & 85.4 & 83.2 & 87.2 & 47 & 22 & 45 & 25 & 73 & 36 \\
\hline DEC-PKT-4 & 84.0 & 89.4 & 88.6 & 91.5 & 45 & 23 & 46 & 24 & 72 & 40 \\
\hline LBL-PKT-4 & 92.0 & 94.2 & 93.2 & 95.0 & 12 & 9 & 13 & 10 & 14 & 12 \\
\hline LBL-PKT-5 & 91.4 & 93.3 & 91.9 & 93.3 & 10 & 9 & 9 & 9 & 15 & 14 \\
\hline FIXWEST1 & 69.7 & 85.3 & 81.6 & 94.3 & 478 & 178 & 480 & 167 & 902 & 312 \\
\hline FIXWEST2 & 72.3 & 85.3 & 83.8 & 95.4 & 351 & 131 & 366 & 171 & 695 & 266 \\
\hline UCB1 & 83.3 & 87.0 & 92.2 & 94.8 & 58 & 48 & 62 & 52 & 106 & 86 \\
\hline UCB2 & 90.0 & 92.4 & 93.8 & 96.1 & 118 & 91 & 90 & 74 & 176 & 134 \\
\hline
\end{tabular}

Table 2: Additional measurements with $\mathrm{X}=5 / \mathrm{Y}=30$ sec. classifier

Because of the way flows are timed out, the maximum number of VC establishments/sec is not equal to the maximum number of VC teardowns/sec for any given trace; however, the average values of these two measurements, although not shown here, are equal. 


\begin{tabular}{|c|c|c|c|}
\hline VC Space & Gateway & Campus/Enterprise Backbone & Internet Backbone \\
\hline $1 \mathrm{~K} \mathrm{VCs}$ & $\begin{array}{l}\text { Clasifier: } X=5 / Y=15 \mathrm{sec} \\
\text { Flow Deletion Delay: } 30-120 \mathrm{sec} \text {. } \\
\text { Expected Performance: } 85 \%\end{array}$ & $\begin{array}{l}\text { Clasifier: } \mathrm{X}=55 \text { / Y = } 5 \mathrm{sec} \\
\text { Flow Deletion Delay: } 30-60 \mathrm{sec} \text {. } \\
\text { Expected Performance: } 51 \%\end{array}$ & $\begin{array}{l}\text { Clasifier: } X=50 / Y=10 \mathrm{sec} . \\
\text { Flow Deletion Delay: } 30 \mathrm{sec} . \\
\text { Expected Performance: } 38 \%\end{array}$ \\
\hline $2 \mathrm{~K} \mathrm{VCs}$ & $\begin{array}{l}\text { Clasifier: } \mathrm{X}=5 \text { / } \mathrm{Y}=60 \mathrm{sec} \\
\text { Flow Deletion Delay: } 30-120 \mathrm{sec} \text {. } \\
\text { Expected Performance: } 90 \%\end{array}$ & $\begin{array}{l}\text { Clasifier: } \mathrm{X}=60 / \mathrm{Y}=50 \mathrm{sec} \\
\text { Flow Deletion Delay: } 30-60 \mathrm{sec} . \\
\text { Expected Performance: } 77 \%\end{array}$ & $\begin{array}{l}\text { Clasifier: } \mathrm{X}=30 / \mathrm{Y}=10 \mathrm{sec} . \\
\text { Flow Deletion Delay: } 30 \mathrm{sec} . \\
\text { Expected Performance: } 47 \%\end{array}$ \\
\hline $8 \mathrm{~K} \mathrm{VCs}$ & $\begin{array}{l}\text { Clasifier: } \mathrm{X}=2 \text { / } \mathrm{Y}=60 \mathrm{sec} \\
\text { Flow Deletion Delay: } 30-120 \mathrm{sec} . \\
\text { Expected Performance: } 93 \%\end{array}$ & $\begin{array}{l}\text { Clasifier: } \mathrm{X}=2 \text { / } \mathrm{Y}=30 \mathrm{sec} \\
\text { Flow Deletion Delay: } 30-60 \mathrm{sec} . \\
\text { Expected Performance: } 96 \%\end{array}$ & $\begin{array}{l}\text { Clasifier: } \mathrm{X}=10 / \mathrm{Y}=10 \mathrm{sec} . \\
\text { Flow Deletion Delay: } 30 \mathrm{sec} . \\
\text { Expected Performance: } 71 \%\end{array}$ \\
\hline $16 \mathrm{~K} \mathrm{VCs}$ & $\begin{array}{l}\text { Clasifier: } \mathrm{X}=2 \text { / } \mathrm{Y}=60 \mathrm{sec} \\
\text { Flow Deletion Delay: } 30-120 \mathrm{sec} \text {. } \\
\text { Expected Performance: } 93 \%\end{array}$ & $\begin{array}{l}\text { Clasifier: } X=2 / Y=60 \mathrm{sec} \\
\text { Flow Deletion Delay: } 30-60 \mathrm{sec} \text {. } \\
\text { Expected Performance: } 96 \%\end{array}$ & $\begin{array}{l}\text { Clasifier: } X=5 / Y=40 \mathrm{sec} . \\
\text { Flow Deletion Delay: } 30 \mathrm{sec} . \\
\text { Expected Performance: } 84 \%\end{array}$ \\
\hline$\infty \mathrm{VCs}$ & $\begin{array}{l}\text { Clasifier: all packets } \\
\text { Flow Deletion Delay: } \infty \\
\text { Expected Performance: } 99 \%\end{array}$ & $\begin{array}{l}\text { Clasifier: all packets } \\
\text { Flow Deletion Delay: } \infty \\
\text { Expected Performance: } 98 \%\end{array}$ & $\begin{array}{l}\text { Clasifier: all packets } \\
\text { Flow Deletion Delay: } \infty \\
\text { Expected Performance: } 97 \%\end{array}$ \\
\hline
\end{tabular}

Table 3: Summary of Recommendataions

arriving datagrams on a new VC are switched and depart before the earlier datagrams from the same flow. Figure 21 shows the percentage of misordered datagrams with respect to the processing time. Notice that to the left of the point of instability, fewer than $0.1 \%$ of datagrams are misordered. As the unstable region is entered, the misordering increases to a peak of about $3.2 \%$, and then drops. This dropping is due to the fact that very few flows are being switched in the first place (see Figure 20), and so there are fewer opportunities for misordered packets. An informal survey of 105 workstations in Stanford University's (non IP Switched) public computing labs revealed that an average of $0.11 \%$ of TCP datagrams arrived out of order. This would indicate that a single IP Switch (operating in the stable region) induces about the same proportion of datagram misordering as conventional networks.

\section{Other Performance Metrics:}

Our study has been using the percentage of datagrams switched as the primary metric, although we have also taken a look at the number of VCs required to support various configurations. There are, of course, other measures that may be of interest in an IP Switching system. For example, it may be desirable to know what proportion of bytes are capable of being switched. Because those flows that carry the most data (and thus tend to have large packet sizes) are often classified as candidates for hardware switching, it seems that the percentage of bytes switched may be higher than the percentage of datagrams switched. Another possible metric is the number of connection setups and teardowns required per second. Some ATM switches are more limited by their capability for connection management than by their capability for total number of established connections, so this connection management overhead may present a bottleneck in some configurations.

Table 2 shows these values for one specific classifier, an $\mathrm{X} / \mathrm{Y}$ classifier with $X=5$ and $Y=30 \mathrm{sec}$. Note that the percentage of bytes switched is higher than the percentage of datagrams switched, as we suggested above. The next two columns show the maximum number of connection setups and the maximum number of connection teardowns in any one second of the simulation. Note that for these columns, we did not include the first one minute of each trace in our analysis, since we did not want to include any transient effects. The last column shows the maximum number of connection management events (setups and teardowns) in any one second, again neglecting the first minute of each trace. The rate of connection management events decreases, similar to the decrease in VC space required, as the classifier is made more strict.

\section{Conclusions}

There are a number of parameters that determine the performance of an IP switch. Some are determined by the switch designer; for example, the number of VCs supported by the switch and the per-packet processing time in the router. Others may be determined by the manager of the switch: the flow classifier and the flow deletion delay. Yet others are determined largely by the environment in which the switch operates; for example, the delay from the time a redirect message is sent until datagrams begin to arrive on the new VC.

We summarize all of our findings by making some specific recommendations for different environments. These are shown in table 3 , and assume that flow type 2 is used (see below) ${ }^{6}$.

As expected, our study shows that the switch designer should aim to support as many VCs as economically viable. Most ATM switches today support between 4096 and 65,536 VCs. Our results indicate that switches supporting at least $10,000 \mathrm{VCs}$ will see acceptable performance with today's traffic. However, the volume and diversity of Internet traffic is growing rapidly. We predict that

${ }^{6}$ These recommendations are based on the set of traces studied. Results will vary from network to network, and these values should be used only as a guide. 
before long, more than $65,536 \mathrm{VCs}$ will be required to provide adequate performance in the core of the Internet. Note that this is the case for flows of type 1 and flows of type 2 .

Our study also shows that a slower router will lead to more misordered datagrams and eventually instability of the IP Switch. However, in all the environments we studied, we found that the router need only forward as few as 3,700 packets per second (assuming flow type 2 ). This is readily achievable today with low-cost general purpose computers.

We found that the $\mathrm{X} / \mathrm{Y}$ classifier works better than either the port or protocol classifiers. It is simple to select values for $\mathrm{X}$ and $\mathrm{Y}$ that lead to over $75 \%$ switched datagrams. When enough VCs are available, our recommended values are: $\mathrm{X}=2$ packets, and $\mathrm{Y}=$ 60 seconds. Note also that the X/Y classifier does not depend on well-known port or protocol numbers; it requires no modifications as different applications come into favor.

Our findings lead us to caution against using large flow deletion delays. These lead to unnecessary exhausting of VCs, and therefore reduce switching performance. We found that a value of 30 seconds suits all of the environments tested.

Finally, our results indicate that the flow creation delay should be kept well below one second. In practice, this should be readily obtained - we expect the delay to be no larger than $20 \mathrm{~ms}$.

We now turn to another decision: should we use flow type 1 or flow type 2? Because the flow type 1 description is a superset of the flow type 2 description, using flow type 2 results in a lower VC usage and a higher switching percentage. This is reflected in our results. In all cases, flow type 2 results in better performance with lower VC usage. Why, then, would anyone use flow type 1 ? When using flow type 2 , all packets from the same source/destination pair are lumped together in the same flow. This is certainly more efficient. However, it does not allow the switch to distinguish different application flows, making it impossible to provide differing classes of service. In reality, we expect that most flows will be classified as type 2, with the network administrator selecting certain specific protocol or port values (i.e. specific high-priority applications) for which to use flow type 1 .

It is clear that the volume of Internet traffic is growing exponentially. We predict that IP switches will soon need in excess of $64 \mathrm{~K}$ VCs to perform well. As the traffic intensity and number of flows increase, it appears that the number of VCs required will also increase; perhaps to the point of overwhelming ATM switches. We can alleviate this problem by using less specific flow descriptions. Using a flow specifier based on IP address prefixes (i.e. aggregated netids) rather than the full 32-bit address should allow multiple type-2 flows to map into one prefix-based flow. This would reduce the number of VCs required, but also require a modification to the IFMP specification.

\section{Acknowledgments}

Steven Lin is supported by a National Science Foundation Graduate Fellowship. Nick McKeown is supported by the Alfred P. Sloan Foundation, a Robert Noyce Faculty Fellowship, and Sumitomo Electric Industries, Inc. We would also like to acknowledge Jeff Mogul of Digital Equipment Corporation for the DECPKT-* traces, Vern Paxson of the Lawrence Berkeley Laboratory for the LBL-PKT-* traces (see [13]), Richard Edell of the University of California, Berkeley for the UCB trace, NLANR for the Fixwest traces, and the members of Ipsilon Networks' technical staff who assisted us with questions about the IFMP protocol.

\section{References}

[1] Peter Newman, Tom Lyon, and Greg Minshall. "Flow Labelled IP: A Connectionless Approach to ATM." Proc. IEEE
Infocom ‘96, vol. 3, pp. 1251-60.

[2] Yakov Rekhter, Bruce Davie, Dave Katz, Eric Rosen, and George Swallow. "Cisco Systems' Tag Switching Architecture Overview.” IETF RFC 2105, Feb. 1997.

[3] Arun Viswanathan, Nancy Feldman, Rick Boivie, and Richard Woundy. "ARIS: Aggregate Route-Based IP Switching.” IETF Internet Draft, March 1997.

[4] K. Nagami, Y. Katsube, Y. Shobatake, A. Mogi, S. Matsuzawa, T. Jinmei, H. Esaki. "Toshiba's Flow Attribute Notification Protocol (FANP) Specification.” IETF RFC 2129, April 1997.

[5] G. Parulkar, D. Schmidt, and J. Turner. "IP/ATM: a Strategy for Integrating IP with ATM.” Proc. ACM SIGCOMM, Cambridge, MA. Sept. 1995.

[6] Ipsilon Networks. "IP Switching: The Intelligence of Routing, the Performance of Switching." White Paper. Available at http://www.ipsilon.com/productinfo/techwp1.html.

[7] W. Doeringer, G. Karjoth, and M. Nassehi. "Routing on Longest-Matching Prefixes.” IEEE/ACM Trans. Networking, Feb. 1996, pp. 86-97.

[8] C. Partridge. "A Fifty Gigabit per Second IP Router." Submitted to IEEE/ACM Trans. Networking.

[9] Nick McKeown, Martin Izzard, Adisak Mekkittikul, William Ellersick, and Mark Horowitz. "The Tiny Tera: A Packet Switch Core.” IEEE Micro Mag., Jan-Feb 1997.

[10] P. Newman, W. L. Edwards, R. Hinden, E. Hoffman, F. Ching Liaw, T. Lyon, and G. Minshall. "Ipsilon Flow Management Protocol Specification for IPv4." IETF RFC 1953, May 1996.

[11] P. Newman, W. L. Edwards, R. Hinden, E. Hoffman, F. Ching Liaw, T. Lyon, and G. Minshall. "Transmission of Flow Labeled IPv4 on ATM Data Links." IETF RFC 1954, May 1996.

[12] Jon Postel. “Internet Protocol.” IETF RFC 791, Sept. 1981.

[13] V. Paxson and S. Floyd. "The Failure of Poisson Modeling." IEEE/ACM Trans. Networking. 3 (3), June 1995, pp. 226244.

[14] H. Che, S. Q. Li, and A. Lin. "Adaptive Resource Management for IP/ATM Hybrid Switching Systems.” Pre-publication manuscript. 